\title{
Fixed points and stability of functional equations in fuzzy ternary Banach algebras
}

\author{
G Asgari ${ }^{1}$, YJ Cho ${ }^{2 *}$, YW Lee ${ }^{3}$ and M Eshaghi Gordji ${ }^{4 *}$
}

"Correspondence: yjcho@gnu.ac.kr;
meshaghi@semnan.ac.ir
${ }^{2}$ Department of Mathematics
Education and the RINS,
Gyeongsang National University,
Chinju, 660-701, Korea
${ }^{4}$ Department of Mathematics,
Semnan University, P.O. Box
35195-363, Semnan, Iran
Full list of author information is
available at the end of the article

\begin{abstract}
By using Diaz and Margolis fixed point theorem, we establish the generalized Hyers-Ulam-Rassias stability of the ternary homomorphisms and ternary derivations between fuzzy ternary Banach algebras associated to the following $(m, n)$-Cauchy-Jensen additive functional equation:

$$
\sum_{\substack{1 \leq i_{1}<\cdots<i_{m} \leq n \\
1 \leq k_{j} \leq n \\
k_{1} \neq j_{j}, \forall j \in\{1, \ldots, m\}}} f\left(\frac{\sum_{j=1}^{m} x_{i_{j}}}{m}+\sum_{l=1}^{n-m} x_{k_{l}}\right)=\frac{(n-m+1)}{n}\left(\begin{array}{c}
n \\
m
\end{array}\right) \sum_{i=1}^{n} f\left(x_{i}\right) .
$$

MSC: $39 \mathrm{~B} 52 ; 46540 ; 26 \mathrm{E} 50$

Keywords: Hyers-Ulam-Rassias stability; Diaz and Margolis contraction theorem; fuzzy ternary Banach algebra; ternary algebras; functional equations
\end{abstract}

\section{Introduction}

A classical question in the theory of functional equations is the following:

When is it true that a function which approximately satisfies a functional equation $\mathcal{E}$ must be close to an exact solution of $\mathcal{E}$ ?

If the problem admits a solution, we say that the equation $\mathcal{E}$ is stable. Such a problem was formulated by Ulam [1] in 1940 and solved in the next year for the Cauchy functional equation by Hyers [2]. Since Hyers, many authors have studied the stability theory for functional equations. The result of Hyers was extended by Aoki [3] in 1950, by considering the unbounded Cauchy differences. Also, Hyers' theorem was generalized by Rassias [4] for linear mappings by considering an unbounded Cauchy difference.

Theorem 1.1 (TM Rassias) Let $f: E \rightarrow E^{\prime}$ be a mapping from a normed vector space $E$ into a Banach space $E^{\prime}$ subject to the following inequality:

$$
\|f(x+y)-f(x)-f(y)\| \leq \epsilon\left(\|x\|^{p}+\|y\|^{p}\right)
$$

for all $x, y \in E$, where $\epsilon$ and $p$ are constants with $\epsilon>0$ and $0 \leq p<1$. Then the limit $L(x)=$ $\lim _{n \rightarrow \infty} \frac{f\left(2^{n} x\right)}{2^{n}}$ exists for all $x \in E$, and $L: E \rightarrow E^{\prime}$ is the unique additive mapping which

\section{Springer}

(c) 2013 Asgari et al.; licensee Springer. This is an Open Access article distributed under the terms of the Creative Commons Attribution License (http://creativecommons.org/licenses/by/2.0), which permits unrestricted use, distribution, and reproduction in any medium, provided the original work is properly cited. 
satisfies

$$
\|f(x)-L(x)\| \leq \frac{2 \epsilon}{2-2^{p}}\|x\|^{p}
$$

for all $x \in E$. Also, iffor each $x \in E$, the function $f(t x)$ is continuous in $t \in \mathbb{R}$, then $L$ is linear.

Găvruta [5] generalized the Rassias' result. Beginning around the year 1980, the stability problems of several functional equations and approximate homomorphisms have been extensively investigated by a number of authors, and there are many interesting results concerning this problem (see [6-29]).

Katsaras [30] defined a fuzzy norm on a vector space to construct a fuzzy vector topological structure on the space. Some mathematicians have defined fuzzy norms on a vector space from various points of view (see [31, 32]). In particular, Bag and Samanta [33], following Cheng and Mordeson [34], gave an idea of a fuzzy norm in such a manner that the corresponding fuzzy metric is of Karmosil and Michalek type [35]. They established a decomposition theorem of a fuzzy norm into a family of crisp norms and investigated some properties of fuzzy normed spaces [36].

Now, we consider a mapping $f: X \rightarrow Y$ satisfying the following functional equation, which is introduced by Rassias and Kim [37] (see also [38]):

$$
\sum_{\substack{1 \leq i_{1}<\ldots<i_{i} \leq n \\
1 \leq k_{l} \leq n \\
k_{l} \neq i_{j}, \forall j \in\{1, \ldots, m\}}} f\left(\frac{\sum_{j=1}^{m} x_{i_{j}}}{m}+\sum_{l=1}^{n-m} x_{k_{l}}\right)=\frac{(n-m+1)}{n}\left(\begin{array}{c}
n \\
m
\end{array}\right) \sum_{i=1}^{n} f\left(x_{i}\right)
$$

for all $x_{1}, \ldots, x_{n} \in X$, where $m, n \in \mathbb{N}$ are fixed integers with $n \geq 2$ and $1 \leq m \leq n$. Especially, we observe that, in the case $m=1$, equation (1.1) yields the Cauchy additive equation

$$
f\left(\sum_{l=1}^{n} x_{k_{l}}\right)=\sum_{l=1}^{n} f\left(x_{i}\right)
$$

Also, we observe that, in the case $m=n$, equation (1.1) yields the Jensen additive equation

$$
f\left(\frac{\sum_{j=1}^{n} x_{j}}{n}\right)=\frac{1}{n} \sum_{l=1}^{n} f\left(x_{i}\right)
$$

Therefore, equation (1.1) is a generalized form of the Cauchy-Jensen additive equation and thus every solution of equation (1.1) may be analogously called the general $(m, n)$-CauchyJensen additive. For the case $m=2$, the authors have established new theorems about the Ulam-Hyers-Rassias stability in quasi- $\beta$-normed spaces [37].

Let $X$ and $Y$ be linear spaces. For each $m$ with $1 \leq m \leq n$, a mapping $f: X \rightarrow Y$ satisfies equation (1.1) for all $n \geq 2$ if and only if $f(x)-f(0)=A(x)$ is Cauchy additive, where $f(0)=0$ if $m<n$. In particular, we have $f((n-m+1) x)=(n-m+1) f(x)$ and $f(m x)=m f(x)$ for all $x \in X$.

Definition 1.1 Let $X$ be a real vector space. A function $N: X \times \mathbb{R} \rightarrow[0,1]$ is called a fuzzy norm on $X$ if for all $x, y \in X$ and $s, t \in \mathbb{R}$, 
(N1) $N(x, t)=0$ for $t \leq 0$;

(N2) $x=0$ if and only if $N(x, t)=1$ for all $t>0$;

(N3) $N(c x, t)=N\left(x, \frac{t}{|c|}\right)$ if $c \neq 0$;

(N4) $N(x+y, s+t) \geq \min \{N(x, s), N(y, t)\}$;

(N5) $N(x, \cdot)$ is a non-decreasing function of $\mathbb{R}$ and $\lim _{t \rightarrow \infty} N(x, t)=1$;

(N6) for any $x \neq 0, N(x, \cdot)$ is continuous on $\mathbb{R}$.

Example 1.1 Let $(X,\|\cdot\|)$ be a normed linear space and $\beta>0$. Then

$$
N(x, t)= \begin{cases}\frac{t}{t+\beta\|x\|}, & t>0, x \in X \\ 0, & t \leq 0, x \in X\end{cases}
$$

is a fuzzy norm on $X$.

Definition 1.2 Let $(X, N)$ be a fuzzy normed vector space. A sequence $\left\{x_{n}\right\}$ in $X$ is said to be convergent if there exists $x \in X$ such that

$$
\lim _{n \rightarrow \infty} N\left(x_{n}-x, t\right)=1
$$

for all $t>0$. In this case, $x$ is called the limit of the sequence $\left\{x_{n}\right\}$ in $X$, which is denoted by $N-\lim _{t \rightarrow \infty} x_{n}=x$.

Definition 1.3 Let $(X, N)$ be a fuzzy normed vector space. A sequence $\left\{x_{n}\right\}$ in $X$ is called a Cauchy sequence if for each $\epsilon>0$ and each $t>0$, there exists $n_{0} \in \mathbb{N}$ such that, for all $n \geq n_{0}$ and $p>0$,

$$
N\left(x_{n+p}-x_{n}, t\right)>1-\epsilon \text {. }
$$

It is well known that every convergent sequence in a fuzzy normed vector space is a Cauchy sequence. If each Cauchy sequence is convergent, then the fuzzy norm is said to be complete and the fuzzy normed vector space is called a fuzzy Banach space.

We say that a mapping $f: X \rightarrow Y$ between fuzzy normed vector spaces $X$ and $Y$ is continuous at a point $x \in X$ if for each sequence $\left\{x_{n}\right\}$ converging to $x_{0} \in X$, the sequence $\left\{f\left(x_{n}\right)\right\}$ converges to $f\left(x_{0}\right)$. If $f: X \rightarrow Y$ is continuous at each $x \in X$, then $f: X \rightarrow Y$ is said to be continuous on $X$ (see [36]).

Ternary algebraic operations were considered in the nineteenth century by several mathematicians such as Cayley [39] who introduced the notion of cubic matrix which in turn was generalized by Kapranov, Gelfand and Zelevinskii in 1990 [40]. The comments on physical applications of ternary structures can be found in [41-45].

Definition 1.4 Let $X$ be a ternary algebra and $(X, N)$ be a fuzzy normed space.

(1) The fuzzy normed space $(X, N)$ is called a ternary fuzzy normed algebra if

$$
N([x y z], s t u) \geq N(x, s) N(y, t) N(z, u)
$$

for all $x, y, z \in X$ and $s, t, u>0$; 
(2) A complete ternary fuzzy normed algebra is called a ternary fuzzy Banach algebra.

Example 1.2 Let $(X,\|\cdot\|)$ be a ternary normed (Banach) algebra. Let

$$
N(x, t)= \begin{cases}\frac{t}{t+\|x\|}, & t>0, x \in X, \\ 0, & t \leq 0, x \in X .\end{cases}
$$

Then $N(x, t)$ is a fuzzy norm on $X$ and $(X, N)$ is a ternary fuzzy normed (Banach) algebra.

Definition 1.5 Let $(X, N)$ and $\left(Y, N^{\prime}\right)$ be two ternary fuzzy normed algebras.

(1) A $\mathbb{C}$-linear mapping $H:(X, N) \rightarrow\left(Y, N^{\prime}\right)$ is called a ternary homomorphism if

$$
H([x y z])=[H(x) H(y) H(z)]
$$

for all $x, y, z \in X$

(2) A $\mathbb{C}$-linear mapping $D:(X, N) \rightarrow(X, N)$ is called a ternary fuzzy derivation if

$$
D([x y z])=[D(x) y z]+[x D(y) z]+[x y D(z)]
$$

for all $x, y, z \in X$.

We apply the following theorem on weighted spaces (see [46-49]).

Theorem 1.2 (The generalized fixed point theorem of Diaz and Margolis) Let $(X, d)$ be $a$ complete metric space and $T: X \rightarrow X$ be a contraction, i.e., there exists $\alpha \in[0,1)$ such that

$$
d(T x, T y) \leq \alpha d(x, y)
$$

for all $x, y \in X$. Then there exists a unique $a \in X$ such that $T a=a$. Moreover, $a=$ $\lim _{n \rightarrow \infty} T^{n} x$ and

$$
d(a, x) \leq \frac{1}{1-\alpha} d(x, T x)
$$

for all $x \in X$.

Throughout this paper, we suppose that $X$ is a ternary fuzzy normed algebra and $Y$ is a ternary fuzzy Banach algebra. Moreover, we assume that $n_{0} \in \mathbb{N}$ is a positive integer and $\mathbb{T}_{\frac{1}{n_{o}}}^{1}:=\left\{e^{i \theta}: 0 \leq \theta \leq \frac{2 \pi}{n_{o}}\right\}$. For the convenience, we use the following abbreviation for a given mapping $f: X \rightarrow Y$ :

$$
\begin{aligned}
& \Delta f\left(x_{1}, \ldots, x_{n}\right) \\
& =\sum_{\substack{1 \leq i_{1}<\cdots<i_{m} \leq n \\
1 \leq k_{l} \leq n \\
k_{l} \neq i, j, \forall j \in\{1, \ldots, m\}}} f\left(\frac{\sum_{j=1}^{m} \mu x_{i_{j}}}{m}+\sum_{l=1}^{n-m} \mu x_{k_{l}}\right)-\frac{(n-m+1)\left(\begin{array}{c}
n \\
m
\end{array}\right) \sum_{i=1}^{n} \mu f\left(x_{i}\right)}{n} .
\end{aligned}
$$




\section{Main results}

In this section, by using the idea of Gavruta and Gavruta [14], we prove the generalized Hyers-Ulam-Rassias stability of ternary homomorphisms related to functional equation (1.1) on ternary fuzzy Banach algebras (see also [50]).

Theorem 2.1 Let $n \geq 3$ and $\varphi: X^{n} \rightarrow[0, \infty)$ be a mapping such that there exists $L<$ $\frac{1}{(n-m+1)^{n-2}}$ such that

$$
\varphi\left(\frac{x_{1}}{n-m+1}, \ldots, \frac{x_{n}}{n-m+1}\right) \leq \frac{L \varphi\left(x_{1}, x_{2}, \ldots, x_{n}\right)}{n-m+1}
$$

for all $x_{1}, \ldots, x_{n} \in X$. Let $f: X \rightarrow Y$ with $f(0)=0$ be a mapping satisfying

$$
N\left(\Delta f\left(x_{1}, \ldots, x_{n}\right), t\right) \geq \frac{t}{t+\varphi\left(x_{1}, \ldots, x_{n}\right)}
$$

and

$$
N(f([a b c])-[f(a) f(b) f(c)], t) \geq \frac{t}{t+\varphi(a, b, c, 0, \ldots, 0)}
$$

for all $\mu \in \mathbb{T}_{\frac{1}{n_{0}}}^{1}, x_{1}, \ldots, x_{n}, a, b, c \in X$ and $t>0$. Then there exists a unique ternary homomorphism $H: X \rightarrow Y$ such that

$$
N(f(x)-H(x), t) \geq \frac{(n-m+1)\left(\begin{array}{c}
n \\
m
\end{array}\right)(1-L) t}{(n-m+1)\left(\begin{array}{l}
n \\
m
\end{array}\right)(1-L) t+L \varphi(x, \ldots, x)}
$$

for all $x \in X$ and $t>0$.

Proof Letting $\mu=1$ and putting $x_{1}=x_{2}=\cdots=x_{n}=x$ in (2.1), we have

$$
N\left(\left(\begin{array}{c}
n \\
m
\end{array}\right) f((n-m+1) x)-\left(\begin{array}{c}
n \\
m
\end{array}\right)(n-m+1) f(x), t\right) \geq \frac{t}{t+\varphi(x, \ldots, x)}
$$

for all $x \in X$ and $t>0$. Set $S_{0}:=\{h: X \rightarrow Y: h(0)=0\}$ and define a mapping $d_{0}: S_{0} \times S_{0} \rightarrow$ $[0, \infty]$ by

$$
d_{0}(f, g)=\inf \left\{\mu \in \mathbb{R}^{+}: N(g(x)-h(x), \mu t) \geq \frac{t}{t+\varphi(x, \ldots, x)}, \forall x \in X, t>0\right\},
$$

where inf $\emptyset=+\infty$. Also, put $S:=\left\{h \in S_{0}: d_{0}(h, f)<\infty\right\}$. Suppose that $d$ is the restriction of $d_{0}$ on $S \times S$. By using the same technique in the proof of Theorem 3.2 [50], we can show that $(S, d)$ is a complete metric space. Now, we define a mapping $J: S \rightarrow S$ by

$$
g(x):=(n-m+1) g\left(\frac{x}{n-m+1}\right)
$$

for all $x \in X$. It is easy to see that $d(J g, J h) \leq L d(g, h)$ for all $g, h \in S$. This implies that

$$
d(f, J f) \leq \frac{L}{(n-m+1)\left(\begin{array}{l}
n \\
m
\end{array}\right)} .
$$


Thus, by Banach's fixed point theorem (Theorem 1.2), $J$ has a unique fixed point $H: X \rightarrow Y$ in $S$ satisfying

$$
H\left(\frac{x}{n-m+1}\right)=\frac{H(x)}{n-m+1}
$$

for all $x \in X$. This implies that $H$ is a unique mapping with (2.5) such that there exists $\mu \in(0, \infty)$ satisfying

$$
N(f(x)-H(x), \mu t) \geq \frac{t}{t+\varphi(x, \ldots, x)}
$$

for all $x \in X$ and $t>0$.

Moreover, we have $d\left(J^{p} f, H\right) \rightarrow 0$ as $p \rightarrow \infty$, which implies

$$
N-\lim _{p \rightarrow \infty} \frac{f\left(\frac{x}{(n-m+1)^{p}}\right)}{(n-m+1)^{-p}}=H(x)
$$

for all $x \in X$. Thus it follows from (2.1) and (2.6) that

$$
\sum_{\substack{1 \leq i_{1}<\cdots<i_{m} \leq n \\
1 \leq k_{l} \leq n \\
k_{l} \neq i j, \forall j \in\{1, \ldots, m\}}} H\left(\frac{\sum_{j=1}^{m} \mu x_{i_{j}}}{m}+\sum_{l=1}^{n-m} \mu x_{k_{l}}\right)=\frac{(n-m+1)}{n}\left(\begin{array}{c}
n \\
m
\end{array}\right) \sum_{i=1}^{n} \mu H\left(x_{i}\right)
$$

for all $\mu \in \mathbb{T}_{\frac{1}{n_{0}}}^{1}$ and $x_{1}, \ldots, x_{n} \in X$. This means that $H: X \rightarrow Y$ is additive. By using the same technique as in the proof of Theorem 2.1 [51], we can show that $H$ is $\mathbb{C}$-linear. On the other hand, by (2.2), we have

$$
N(\alpha, \beta) \geq \frac{t}{t+\varphi\left(\frac{a}{(n-m+1)^{p}}, \frac{b}{(n-m+1)^{p}}, \frac{c}{(n-m+1)^{p}}, 0,0, \ldots, 0\right)}
$$

for all $a, b, c \in X$ and $t>0$, where

$$
\begin{aligned}
& \alpha=\frac{f\left(\frac{[a b c]}{(n-m+1)^{(n-1) p}}\right)}{(n-m+1)^{-(n-1) p}}-\frac{\left[f\left(\frac{a}{(n-m+1)^{p}}\right) f\left(\frac{b}{(n-m+1)^{p}}\right) f\left(\frac{c}{(n-m+1)^{p}}\right)\right]}{(n-m+1)^{-(n-1) p}}, \\
& \beta=\frac{t}{(n-m+1)^{-(n-1) p}} .
\end{aligned}
$$

Then we have, as $p \rightarrow+\infty$,

$$
\begin{aligned}
& N\left(\frac{f\left(\frac{[a b c]}{(n-m+1)^{(n-1) p}}\right)}{(n-m+1)^{-(n-1) p}}-\frac{\left[f\left(\frac{a}{(n-m+1)^{p}}\right) f\left(\frac{b}{(n-m+1)^{p}}\right) f\left(\frac{c}{(n-m+1)^{p}}\right)\right]}{(n-m+1)^{-(n-1) p}}, t\right) \\
& \quad \geq \frac{\frac{t}{(n-m+1)^{(n-1) p}}}{\frac{t}{(n-m+1)^{(n-1) p}}+\varphi\left(\frac{a}{(n-m+1)^{p}}, \frac{b}{(n-m+1)^{p}}, \frac{c}{(n-m+1)^{p}}, 0,0, \ldots, 0\right)} \\
& \quad \geq \frac{\frac{t}{(n-m+1)^{(n-1) p}}}{\frac{t}{(n-m+1)^{(n-1) p}}+\frac{L^{p} \varphi(a, b, c, 0,0, \ldots, 0)}{(n-m+1)^{p}}} \rightarrow 1
\end{aligned}
$$


for all $a, b, c \in X$ and $t>0$. So, it follows that

$$
N(H([a b c])-[H(a) H(b) H(c)], t)=1
$$

for all $a, b, c \in X$ and $t>0$. Hence we have $H([a b c])=[H(a) H(b) H(c)]$ for all $a, b, c \in X$. This means that $H$ is a ternary homomorphism. This completes the proof.

Theorem 2.2 Let $\varphi: X^{n} \rightarrow[0, \infty)$ be a mapping such that there exists $L<1$ with

$$
\varphi\left(x_{1}, \ldots, x_{n}\right) \leq(n-m+1) L \varphi\left(\frac{x_{1}}{n-m+1}, \ldots, \frac{x_{n}}{n-m+1}\right)
$$

for all $x_{1}, x_{2}, \ldots, x_{n} \in X$. Let $f: X \rightarrow Y$ be a mapping with $f(0)=0$ satisfying (2.1). Then the limit $H(x):=N-\lim _{p \rightarrow \infty} \frac{f\left((n-m+1)^{p} x\right)}{(n-m+1)^{p}}$ exists for all $x \in X$ and $H: X \rightarrow Y$ is defined as a ternary homomorphism such that

$$
N(f(x)-H(x), t) \geq \frac{(n-m+1)\left(\begin{array}{l}
n \\
m
\end{array}\right)(1-L) t}{(n-m+1)\left(\begin{array}{c}
n \\
m
\end{array}\right)(1-L) t+\varphi(x, \ldots, x)}
$$

for all $x \in X$ and $t>0$.

Proof Let $(S, d)$ be the metric space defined as in the proof of Theorem 2.1. Consider the mapping $T: S \rightarrow S$ defined by $\operatorname{Tg}(x):=\frac{g((n-m+1) x)}{n-m+1}$ for all $x \in X$. One can show that $d(g, h)=\epsilon$ implies that $d(T g, T h) \leq L \epsilon$ for all positive real numbers $\epsilon$. This means that $T$ is a contraction on $(S, d)$. The mapping

$$
H(x):=N-\lim _{p \rightarrow \infty} \frac{f\left((n-m+1)^{p} x\right)}{(n-m+1)^{p}}
$$

is the unique fixed point of $T$ in $S$ and $H$ has the following property:

$$
(n-m+1) H(x)=H((n-m+1) x)
$$

for all $x \in X$. This implies that $H$ is a unique mapping satisfying (2.8) such that there exists $\mu \in(0, \infty)$ satisfying $N(f(x)-H(x), \mu t) \geq \frac{t}{t+\varphi(x, \ldots, x)}$ for all $x \in X$ and $t>0$.

The rest of the proof is similar to the proof of Theorem 2.1. This completes the proof.

Now, we investigate the Hyers-Ulam-Rassias stability of ternary derivations in ternary fuzzy Banach algebras.

Theorem 2.3 Let $X$ be a fuzzy Banach algebra. Let $\varphi: X^{n} \rightarrow[0, \infty)$ be a function such that there exists $L<\frac{1}{(n-m+1)^{n-2}}$ with

$$
\varphi\left(\frac{x_{1}}{n-m+1}, \ldots, \frac{x_{n}}{n-m+1}\right) \leq \frac{L \varphi\left(x_{1}, x_{2}, \ldots, x_{n}\right)}{n-m+1}
$$

for all $x_{1}, \ldots, x_{n} \in X$. Let $f: X \rightarrow X$ be a mapping with $f(0)=0$ satisfying (2.1) and

$$
N(f([a b c])-[f(a) b c]-[a f(b) c]-[a b f(c)], t) \geq \frac{t}{t+\varphi(a, b, c, 0,0, \ldots, 0)}
$$


for all $a, b, c \in X$ and $t>0$. Then $D(x):=N-\lim _{p \rightarrow \infty} \frac{f\left(\frac{x}{(n-m+1)^{p}}\right)}{(n-m+1)^{-p}}$ exists for all $x \in X$ and $D:$ $X \rightarrow X$ is defined as a unique ternary derivation such that

$$
N(f(x)-D(x), t) \geq \frac{(n-m+1)\left(\begin{array}{c}
n \\
m
\end{array}\right)(1-L) t}{(n-m+1)\left(\begin{array}{l}
n \\
m
\end{array}\right)(1-L) t+L \varphi(x, \ldots, x)}
$$

for all $x \in X$ and $t>0$.

Proof By the same reasoning as that in the proof of Theorem 2.1, the mapping $D: X \rightarrow X$ is a unique $\mathbb{C}$-linear mapping which satisfies (2.10).

Now, we show that $D$ is a ternary derivation. By (2.9), we have

$$
\begin{aligned}
& N\left(\frac{f\left(\frac{[a b c]}{(n-m+1)^{(n-1) p}}\right)}{(n-m+1)^{-(n-1) p}}-\frac{\left[f\left(\frac{a}{(n-m+1)^{p}}\right) b c\right]-\left[a f\left(\frac{b}{(n-m+1)^{p}}\right) c\right]-\left[a b f\left(\frac{c}{(n-m+1)^{p}}\right)\right]}{(n-m+1)^{-(n-1) p}},\right. \\
& \left.\quad \frac{t}{(n-m+1)^{-(n-1) p}}\right) \\
& \quad \geq \frac{t}{t+\varphi\left(\frac{a}{(n-m+1)^{p}}, \frac{b}{(n-m+1)^{p}}, \frac{c}{(n-m+1)^{p}}, 0,0, \ldots, 0\right)}
\end{aligned}
$$

for all $a, b, c \in X$ and $t>0$. Then we have

$$
\begin{aligned}
& N\left(\frac{f\left(\frac{[a b c]}{(n-m+1)^{(n-1) p}}\right)}{(n-m+1)^{-(n-1) p}}-\frac{\left[f\left(\frac{a}{(n-m+1)^{p}}\right) b c\right]-\left[a f\left(\frac{b}{(n-m+1)^{p}}\right) c\right]-\left[a b f\left(\frac{c}{(n-m+1)^{p}}\right)\right]}{(n-m+1)^{-(n-1) p}}, t\right) \\
& \quad \geq \frac{\frac{t}{(n-m+1)^{(n-1) p}}}{\frac{t}{(n-m+1)^{(n-1) p}}+\varphi\left(\frac{a}{(n-m+1)^{p}}, \frac{b}{(n-m+1)^{p}}, \frac{c}{(n-m+1)^{p}}, 0,0, \ldots, 0\right)} \\
& \geq \frac{\frac{t}{(n-m+1)^{(n-1) p}}}{\frac{t}{(n-m+1)^{(n-1) p}}+\frac{L^{p} \varphi(a, b, c, 0,0, \ldots, 0)}{(n-m+1)^{p}}} \rightarrow 1 \quad \text { when } p \rightarrow+\infty
\end{aligned}
$$

for all $a, b, c \in X$ and $t>0$. So, we have

$$
N(D([a b c])-[D(a) b c]-[a D(b) c]-[a b H(c)], t)=1
$$

for all $a, b, c \in X$ and $t>0$. Hence we have $D([a b c])=[D(a) b c]+[a D(b) c]+[a b D(c)]$ for all $a, b, c \in X$. This means that $D$ is a ternary derivation. This completes the proof.

Theorem 2.4 Let $X$ be a fuzzy Banach algebra. Let $\varphi: X^{n} \rightarrow[0, \infty)$ be a function such that there exists $L<1$ with

$$
\varphi\left(x_{1}, \ldots, x_{n}\right) \leq(n-m+1) L \varphi\left(\frac{x_{1}}{n-m+1}, \ldots, \frac{x_{n}}{n-m+1}\right)
$$

for all $x_{1}, x_{2}, \ldots, x_{n} \in X$. Let $f: X \rightarrow X$ be a mapping with $f(0)=0$ satisfying (2.1) and (2.9). Then the limit $D(x):=N-\lim _{p \rightarrow \infty} \frac{f\left((n-m+1)^{p} x\right)}{(n-m+1)^{p}}$ exists for all $x \in X$ and $D: X \rightarrow X$ is defined as a ternary derivation such that

$$
N(f(x)-D(x), t) \geq \frac{(n-m+1)\left(\begin{array}{c}
n \\
m
\end{array}\right)(1-L) t}{(n-m+1)\left(\begin{array}{l}
n \\
m
\end{array}\right)(1-L) t+\varphi(x, \ldots, x)}
$$

for all $x \in X$ and $t>0$. 


\section{Competing interests}

The authors declare that they have no competing interests.

\section{Authors' contributions}

All authors read and approved the final manuscript.

\section{Author details}

${ }^{1}$ Department of Mathematics, Islamic Azad University, Aligoudarz Branch, Aligoudarz, Iran. ${ }^{2}$ Department of Mathematics Education and the RINS, Gyeongsang National University, Chinju, 660-701, Korea. ${ }^{3}$ Department of Computer Hacking and Information Security, Daejeon University, Daejeon, 300-716, Korea. ${ }^{4}$ Department of Mathematics, Semnan University, P.O. Box 35195-363, Semnan, Iran.

\section{Acknowledgements}

The second author of this work was partially supported by Basic Science Research Program through the National Research Foundation of Korea (NRF) funded by the Ministry of Education, Science and Technology (Grant No. 2012-0008170).

\section{Received: 22 October 2012 Accepted: 27 January 2013 Published: 10 April 2013}

\section{References}

1. Ulam, SM: A Collection of the Mathematical Problems. Interscience, New York (1960)

2. Hyers, DH: On the stability of the linear functional equation. Proc. Natl. Acad. Sci. USA 27, $222-224$ (1941)

3. Aoki, T: On the stability of the linear transformation in Banach spaces. J. Math. Soc. Jpn. 2, 64-66 (1950)

4. Rassias, TM: On the stability of the linear mapping in Banach spaces. Proc. Am. Math. Soc. 72, $297-300$ (1978)

5. Găvruta, P: A generalization of the Hyers-Ulam-Rassias stability of approximately additive mappings. J. Math. Anal. Appl. 184, 431-436 (1994)

6. Bourgin, DG: Classes of transformations and bordering transformations. Bull. Am. Math. Soc. 57, 223-237 (1951)

7. Cădariu, L, Radu, V: Fixed points and the stability of Jensen's functional equation. J. Inequal. Pure Appl. Math. 4(1), Article ID 4 (2003)

8. Cădariu, L, Radu, V: Fixed points and the stability of quadratic functional equations. An. Univ. Timişoara, Ser. Mat. Inform 41, 25-48 (2003)

9. Czerwik, S: The stability of the quadratic functional equation. In: Rassias, TM Tabor, J (eds.) Stability of Mappings of Hyers-Ulam Type, pp. 81-91. Hadronic Press, Florida (1994)

10. Eshaghi Gordji, M, Rassias, JM, Ghobadipour, N: Generalized Hyers-Ulam stability of generalized (N, K)-derivations. Abstr. Appl. Anal. 2009, Article ID 437931 (2009)

11. Eshaghi Gordji, M, Ghaemi, MB, Kaboli Gharetapeh, S, Shams, S, Ebadian, A: On the stability of J*-derivations. J. Geom. Phys. 60, 454-459 (2010)

12. Eshaghi Gordji, M, Najati, A: Approximately J*-homomorphisms: a fixed point approach. J. Geom. Phys. 60, 809-814 (2010)

13. Rassias, JM, Jun, KW, Kim, HM: Approximate $(m, n)$-Cauchy-Jensen additive mappings in $C^{*}$-algebras. Acta Math. Sin. 27(10), 1907-1922 (2011)

14. Găvruta, P, Găvruta, L: A new method for the generalized Hyers-Ulam-Rassias stability. Int. J. Nonlinear Anal. Appl. 1, 11-18 (2010)

15. Khodaei, H, Rassias, TM: Approximately generalized additive functions in several variables. Int. J. Nonlinear Anal. Appl. 1, 22-41 (2010)

16. Cholewa, PW: Remarks on the stability of functional equations. Aequ. Math. 27, 76-86 (1984)

17. Czerwik, S: On the stability of the quadratic mapping in normed spaces. Abh. Math. Semin. Univ. Hamb. 62, 239-248 (1992)

18. Saadati, R, Vaezpour, M, Cho, YJ: A note to paper 'On the stability of cubic mappings and quartic mappings in random normed spaces'. J. Inequal. Appl. 2009, Article ID 214530 (2009)

19. Saadati, R, Zohdi, MM, Vaezpour, SM: Nonlinear L-random stability of an ACQ functional equation. J. Inequal. Appl. 2011, Article ID 194394 (2011)

20. Azadi Kenary, H, Rezaei, H, Ghaffaripour, A, Talebzadeh, S, Park, C, Lee, JR: Fuzzy Hyers-Ulam stability of an additive functional equation. J. Inequal. Appl. 2011, 140 (2011)

21. Park, C, Lee, JR, Rassias, TM, Saadati, R: Fuzzy*-homomorphisms and fuzzy *-derivations in induced fuzzy C*-algebras Math. Comput. Model. 54, 2027-2039 (2011)

22. Park, C, Eshaghi Gordji, M, Cho, YJ: Stability and superstability of generalized quadratic ternary derivations on non-Archimedean ternary Banach algebras: a fixed point approach. Fixed Point Theory Appl. 2012, 97 (2012)

23. Cho, YJ, Kang, SM, Sadaati, R: Nonlinear random stability via fixed-point method. J. Appl. Math. 2012, Article ID $902931(2012)$

24. Cho, YJ, Park, C, Saadati, R: Functional inequalities in non-Archimedean Banach spaces. Appl. Math. Lett. 23 1238-1242 (2010)

25. Cho, YJ, Saadati, R, Vahidi, J: Approximation of homomorphisms and derivations on non-Archimedean Lie $C^{*}$-algebras via fixed point method. Discrete Dyn. Nat. Soc. 2012, Article ID 373904 (2012)

26. Saadati, R, Cho, YJ, Vahidi, J: The stability of the quartic functional equation in various spaces. Comput. Math. Appl. 60 1994-2002 (2010)

27. Saadati, R, Park, C: Non-Archimedean L-fuzzy normed spaces and stability of functional equations. Comput. Math Appl. 60, 2488-2496 (2010)

28. Saadati, R, Vaezpour, SM, Park, C: The stability of the cubic functional equation in various spaces. Math. Commun. 16, 131-145 (2011)

29. Rassias, TM: On the stability of functional equations in Banach spaces. J. Math. Anal. Appl. 251, $264-284$ (2000)

30. Katsaras, AK: Fuzzy topological vector spaces. Fuzzy Sets Syst. 12, 143-154 (1984)

31. Felbin, C: Finite-dimensional fuzzy normed linear space. Fuzzy Sets Syst. 48, 239-248 (1992) 
32. Park, C: Fuzzy stability of a functional equation associated with inner product spaces. Fuzzy Sets Syst. 160, 1632-1642 (2009)

33. Bag, T, Samanta, SK: Finite dimensional fuzzy normed linear spaces. J. Fuzzy Math. 11, 687-705 (2003)

34. Cheng, SC, Mordeson, JN: Fuzzy linear operators and fuzzy normed linear spaces. Bull. Calcutta Math. Soc. 86, 429-436 (1994)

35. Karmosil, I, Michalek, J: Fuzzy metric and statistical metric spaces. Kybernetica 11, 326-334 (1975)

36. Bag, T, Samanta, SK: Fuzzy bounded linear operators. Fuzzy Sets Syst. 151, 513-547 (2005)

37. Rassias, JM, Kim, H: Generalized Hyers-Ulam stability for general additive functional equations in quasi $\beta$-normed spaces. J. Math. Anal. Appl. 356, 302-309 (2009)

38. Azadi Kenary, $\mathrm{H}$ : Non-Archimedean stability of Cauchy-Jensen type functional equation. J. Nonlinear Anal. Appl. 1, 1-10 (2011)

39. Cayley, A: On the 34 concomitants of the ternary cubic. Am. J. Math. 4, 1-15 (1981)

40. Kapranov, M, Gelfand, IM, Zelevinskii, A: Discrimininants, Resultants and Multidimensional Determinants. Birkhauser, Berlin (1994)

41. Abramov, V, Kerner, R, Liivapuu, O, Shitov, S: Algebras with ternary law of composition and their realization by cubic matrices. J. Gen. Lie Theory Appl. 3, 77-94 (2009)

42. Kerner, R: Ternary and non-associative structures. Int. J. Geom. Methods Mod. Phys. 5, 1265-1294 (2008)

43. Kerner, R: The cubic chessboard. Geometry and physics. Class. Quantum Gravity 14, 203-225 (1997)

44. Sewell, GL: Quantum Mechanics and Its Emergent Macrophysics. Princeton University Press, Princeton (2002)

45. Zettl, H: A characterization of ternary rings of operators. Adv. Math. 48, 117-143 (1983)

46. Diaz, J, Margolis, B: A fixed point theorem of the alternative for contractions on a generalized complete metric space. Bull. Am. Math. Soc. 74, 305-309 (1968)

47. Mihet, D, Radu, V: On the stability of the additive Cauchy functional equation in random normed spaces. J. Math. Anal. Appl. 343, 567-572 (2008)

48. Park, C, Lee, JR, Rassias, TM, Saadati, R: Fuzzy*-homomorphisms and fuzzy *-derivations in induced fuzzy C*-algebras Math. Comput. Model. 54, 2027-2039 (2011)

49. Radu, V: The fixed point alternative and the stability of functional equations. Fixed Point Theory 4, 91-96 (2003)

50. Eshaghi Gordji, M, Moradlou, F: Approximate Jordan derivations on Hilbert C*-moduls. Fixed Point Theory (to appear)

51. Eshaghi Gordji, M: Nearly involutions on Banach algebras; a fixed point approach. Fixed Point Theory 12, 341-348 (2011)

doi:10.1186/1029-242X-2013-166

Cite this article as: Asgari et al.: Fixed points and stability of functional equations in fuzzy ternary Banach algebras. Journal of Inequalities and Applications 2013 2013:166.

\section{Submit your manuscript to a SpringerOpen ${ }^{\odot}$ journal and benefit from:}

- Convenient online submission

Rigorous peer review

- Immediate publication on acceptance

- Open access: articles freely available online

- High visibility within the field

- Retaining the copyright to your article 